\title{
An Energy Efficient Architecture of IoT Based on Service Oriented Architecture (SOA)
}

\author{
Sudhanshu Maurya \\ Department of Computer Science \& IT, Jharkhand Rai University, Ranchi, Jharkhand, India \\ E-mail: sam.sudhanshu@gmail.com, ${ }^{1}$ WebAddress: www.jru.edu.in \\ Dr. Kuntal Mukherjee \\ Department of Computer Science \& Engineering, BIT Mesra, Ranchi, Jharkhand, India \\ E-mail: kmukherjee@bitmesra.ac.in, ${ }^{2}$ WebAddress: www.bitmesra.ac.in
}

Keywords: cloud, IoT, energy Efficient, architecture, SOA

Received: August 25, 2017

\begin{abstract}
In the present scenario, IoT is a platform where everyday gadgets are becoming smarter, each day processing will become shrewd, and every day verbal exchange is becoming informative. Even as the IoT is still searching for its basic form, its result has already started in making exquisite strides as a universal solution media for the associated situation. The study which focused on architecture always paves the conformation of associated discipline. The shortage of overall architectural abilities is at this time resisting the researchers to get a way of the scope of procedures based on energy efficient IoT. In this endeavor Service Oriented Architecture (SOA) based IoT architecture and algorithm have been proposed to address energy efficiency in IoT environment. Experimental result has been shown to lay bare the effectiveness of the proposed approach.
\end{abstract}

Povzetek: V tem rokopisu je bila predlagana energetsko učinkovita arhitektura interneta stvari na osnovi storitveno usmerjene arhitekture (SOA) z algoritmom.

\section{Introduction}

Internet of Things (IoT) is currently turning into a novel upheaval after the internet in information and communication industry. It is the mixture of innovation of internet and hardware equipment, which is able to receive and send the signals. The fundamental thought of this idea is an unavoidable availability of things near us. A spread of factors and articles like Sensors [1], Smartphones, Radio-Frequency Identification (RFID) tags [2], actuators and so on through one of a kind addressing schemes, are capable of engaging with one another and coordinate with their friends to reach commonplace desires [3]. By end of the year 2020, total number of different inter-connected devices will reach on IoT network into several billion. Devices may be actuators, mobile phones, laptops, sensors, appliances used in the office or home and other nodes that can be actuated or connected [4]. There is numerous bridge credibility among IoT and distinctive technologies which incorporate senor, communication, SCM, storage, cloud computing, visualization and mining of data, and its miles generally used in warehousing and logistics, medicine and healthcare, acumen and amusing ambiance [5]. Owing to the fast improvement of IoT, numerous associations and manufacturers resolve to search for out procedures that make the IoT more conventional. For an ample duration of time, IoT will remain at the theoretical level. As it may, get to be distinctly indicated in the IoT planned research roadmap of Europe, devices like cellular gadgets and Machine to Machine (M2M) or Things to Things (T2T), can be primary leaders for the advance development of IoT [6]. The cachet and gesture of an article can be detected with the help of sensor (like RFID) in special condition [7]. For example, take a container, with the help of RFID gadget, the sensor can identify it as a teacup, cup or glass [8]. We likewise know that it had been lifted gradually with the help of a sensor on the container we are able to receive the message like "fluid of glass is excessively warm, making it difficult to drink". The pertinence of syntactic research with regards to the IoT was addressed by authors in [6]. Where substances ontologies may want to offer advantages in institutionalization (managing associations among heterogeneous gadgets and information suppliers), thing disclosure and pursuit (where metadata and semantic comments are profoundly important), or interoperability (particularly tended to by advances in IoT-particular semantic innovations) [9] and additionally "semantically determined code era of gadget interfaces". The purpose of this manuscript is to deliberate the basic concept of IoT and propose a cloud-centric view of IoT framework and new energy efficient architecture of IoT based on SOA (Service Oriented Architecture).

SOA is basically a pool of services, which are able to communicate mutually. Here communication includes the passing of data or managing the activities. Sometimes, connecting between services is desirable.

Section 1 discusses the introductory part of IoT, then in section 2 literature review has been done which includes the architecture of IoT and IP based connectivity 
architecture of IoT. In Section 3, we have proposed an energy efficient IoT architecture based on SOA with the algorithm. In Section 4, the experimental setup and result have been shown and section 5 has given conclusion of the manuscript.

\section{Literature review}

IoT is a brand new archetype through which commonly recognizable things are virtually characterized, which will allow in establishing the communication with them. Originally IoT was invented in the year of 1999, its miles nevertheless in its beginning in development unexpectedly to 26 billion gadgets in 2020 [10]. The perception and prescient of the ERC placed the IoT in relation to an arrangement of innovation empowering agents where we can recognize knowledge, segment or morphological advancements [6]. Some portion of the upcoming technologies of the internet, IoT is thrice - A worldview which is Anytime, Anything and Anywhere, that is progressively turning into the best components of the the Internet [5]. It permitting "humans and things to be related preferably the use of any route, any network and any carrier" in a six - A connectivity paradigm as shown in Figure 1 [11].

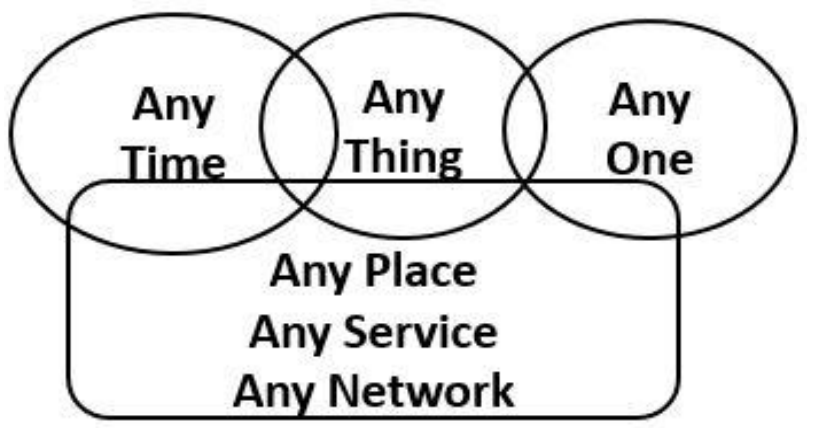

Figure 1: The Six - A Connectivity of IoT

The majority of the assets required in the IoT system are trading data utilizing an extensive variety of communicable hardware and in some of the scenarios, the sources' existence cycle is certain to the battery lifestyles [12]. Along with these, a component for designing the system hardware progressively could enhance the operational time of the gadgets and set up imperative connections by the approach of Green ICT. To overcome this issue an automated many-operator framework is discussed in the research work of [13], which influencing system demonstrations and enhancing the functioning time of gadgets. To ensure interoperability of entire systems, research work is done by authors in [14], [15], [16] concentrated using the idea of SOA directly on devices. In regards to sensor networks, energy proficient data spread had been researched. Authors in their research work, created different models for components of cloud computing environment like communication, computation and storage device [17]. An area assisted methodology is projected with the help of a protocol that supports the situation data to scale back repetitive transmissions of data [18]. Work was done in [19], [20], authors focus on demand allocation of resources which used prediction algorithm for forecasting of resources which are going to be used. In the research work of [21] authors proposed a system that manages complexity and changes consequently in the IoT environment. To accomplish this objective, authors have proposed a framework incorporates the accompanying developments which include dependable, strong, adaptable, expandable and object-oriented layouts to encourage interfaces and reuses of framework segments. Making a cosmology for IoT is a troublesome deed to accomplish as it included a wide range of pitches [22]. Moreover, ontologies which are associated with statistic figures, data received from sensors and different statistics generating gadgets should be taken into consideration so that it will have the ability if you want to work approximate data. For minimum consumption of energy authors [23] in their research work, using few servers in place of multiple servers and distribute the tasks to various virtual machines inside the server of the cloud environment. Authors [24] in their research work projected an algorithm that backs transmission of spatial information and process inquiries in light of their area. Another research work in [25] tended to energy potency in information scattering for IoT. For the minimization of energy consumption, authors [26] in their research work proposed a scheduling algorithm. To provide energy efficient job allocation and load balancing authors [27] proposed a scheduling algorithm named eSTAB for data centers.

\subsection{Architecture of IoT}

IoT architecture comprises of different layers, shown in Figure 2.

\begin{tabular}{|c|c|c|c|}
\hline Application Layer \\
\hline \multicolumn{3}{|c|}{$\uparrow$ Network Layer } \\
\begin{tabular}{|c|c|c|c|}
\hline Mobile Network & Internet & $\begin{array}{c}\text { Information } \\
\text { Network }\end{array}$ \\
\hline \multicolumn{4}{|c|}{ Sensing Layer } \\
\hline RFID & Sensor Network & $\cdots \cdots$ & GPS \\
\hline
\end{tabular}
\end{tabular}

Figure 2: Architecture of IoT [28].

The lowermost layer that is sensing layer, comprises of different sensing devices. The information that is processed by these sensing devices is linked with different applications present at the topmost layer - application layer through the cloud computing [28]. Thus, a cloudcentric hallucination is the utmost need in order to employ IoT extensively.

Obviously, the whole IoT technology pivots on the lowermost layer that is sensing layer. The sensing layer comprises RFID (Radio - Frequency Identification), sensor network and Global Positioning System (GPS) [28]. Thus in the epoch of IoT, all objects and devices are interconnected and interact with each other [29]. Cloud computing platform is built over the different components of the middle layer namely mobile networks, Internet 
backbone, and the various information networks. A cloudcentric view of IoT framework is depicted in Figure 3.

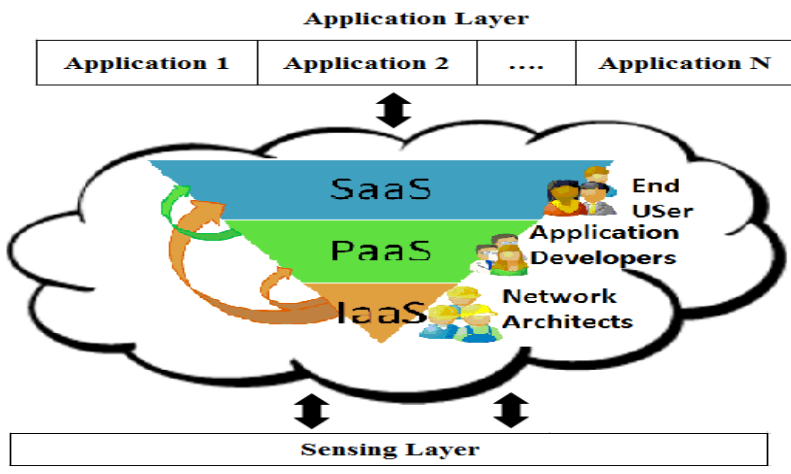

Figure 3: Cloud Centric view of IoT Framework.

Further, for an effective communication among the objects and devices in IoT, unique IP address is the utmost need for all objects. This unique IP address is provided by IPv6 protocol used in IoT. In order to distinguish all computers and pervasive devices, the IPv6 protocol provides 2128 IP addresses [28]. Further, three types of communications exist in IoT, which are $\mathrm{H} 2 \mathrm{H}, \mathrm{H} 2 \mathrm{~T}$, and $\mathrm{T} 2 \mathrm{~T}$, where $\mathrm{H}$ stands for Human, $\mathrm{T}$ stands for Things.

\subsection{IP based connectivity architecture of IoT}

It has been observed that the building block of IoT network comprises of smart object network and the Internet. The Internet is based on TCP/IP architecture and each machine on the Internet has a unique IP address [30]. Now, it's a big question, what would be the architecture for a smart object network? What would be the addressing scheme for it? Obviously, for the effective design of IoT, the first step will be assigning a unique address to all the things surrounding us including all computers and pervasive devices [28]. Due to interoperability across devices, scalability, and ease of deployment ability features of IP architecture, it is a natural choice to adopt in order to develop smart object network [31].

Nowadays, the wireless sensor network community has widely accepted IP architecture to establish wireless sensor network system - the largest subset of sensing network [32]. Furthermore, the interoperability with existing systems characteristic of IP architecture has lead to evolve IP-based sensor network system [33]. The overall IoT technology integrates a variety of legitimate heterogeneity in the used networking technologies with the smart object networking [5]. The smart object networking comprises of cellular, sensor network, RFID etc., which are different from each other [8]. To bind all such heterogeneities together, IP communications is best suited, shown in Figure 4. IP plays the central role for all types of communication irrespective of their nature. All the network applications depend on IP.

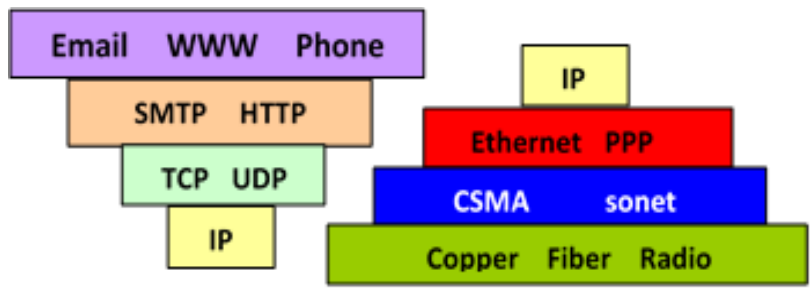

Figure 4: IP-based Communication Model.

Hence, we can say that IP-based connectivity model is best suited in designing IoT network [30]. After assigning unique IP addresses to all devices, the next question is to design an energy efficient network of these devices and hence IoT, which has been discussed in the subsequent sections.

\section{Proposed energy efficient design of IoT algorithm based on SOA}

It is obvious that when the sensor nodes are active then they consume more energy but when they are in the dormant state then they consume less energy. For this endeavor, the random geometric graph concept has been used [34]. A link, hence energy consumption, is established between two sensor nodes when they are very close to each other. For this, different sensor nodes are arranged according to the Poisson point process that is

$$
\frac{e^{-\lambda} \lambda^{x}}{x !}
$$

Where $x$ is random variable and $\lambda$ is parameter of the distribution. Here as the value of $\lambda$ increases more and more nodes exists that are close to each other and start to communicate mutually, in other words, they become active. Hence, it is the utmost need to find out the critical value of $\lambda$, say, $\lambda_{c}$ for which only the required number of sensor nodes would be made active and rest would be made in the dormant state. Thus the proposed Hypothesis and the proposed algorithm for the same are discussed in next sub sections.

\subsection{Hypothesis}

The hypothesis of proposed algorithm is given below: Hypothesis 1: The sensor nodes are arranged as per the Poisson point process, that is, $\frac{e^{-\lambda} \lambda^{x}}{x !}$

Here, $x$ is the random variable, $\lambda$ is known as a parameter of the distribution, $e$ is exponential. Obviously, $\lambda=n p$ where $n$ is number of sensor nodes and $p$ represents the probability of finding them in correct order.

\subsection{SOA for IoT}

The SOA-based architecture of IoT should be discussed in view of security, Quality of Service (QoS), energy management, infrastructure monitoring etc. Out of these 
parameters, here we only focus on the energy management and QoS aspects.

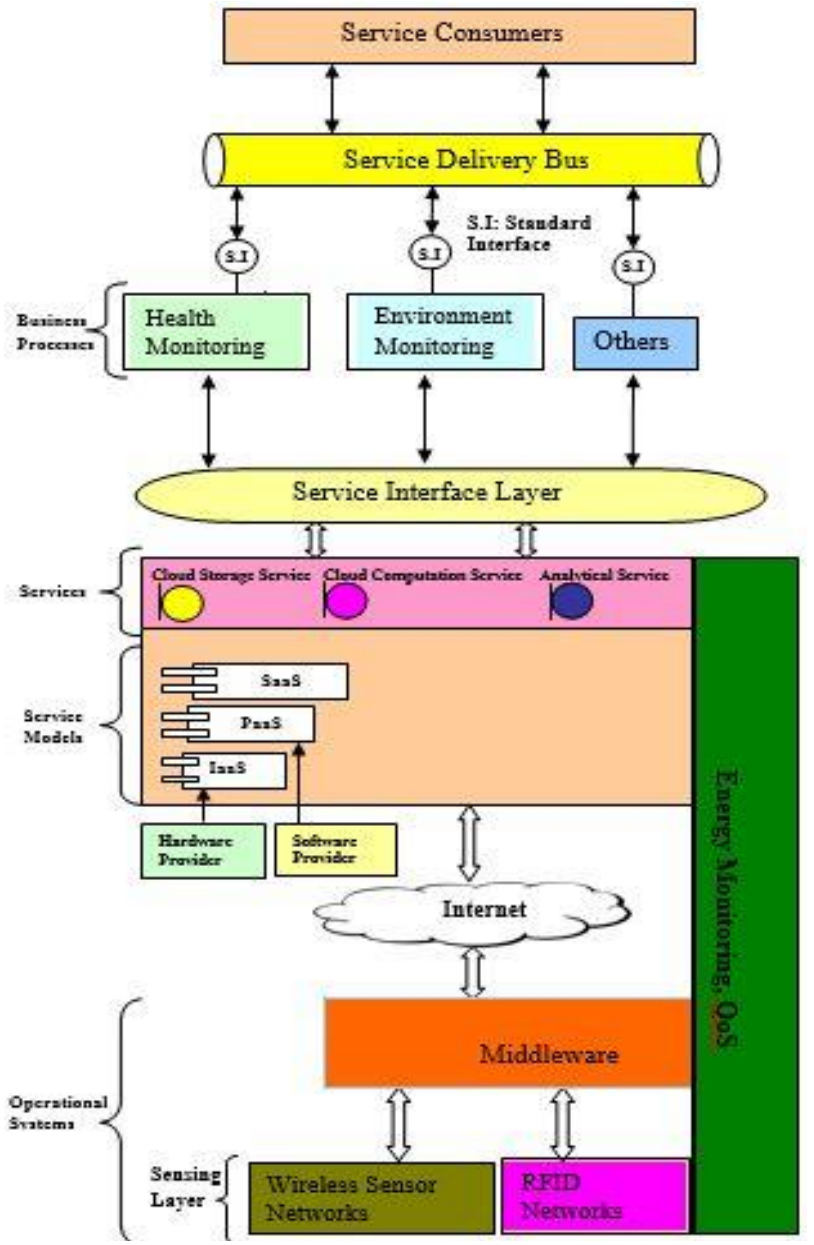

Figure 5: Proposed Energy efficient IoT Architecture based on SOA.

In the proposed architecture, the raw data captured by the sensing layer which comprises of RFID and WSN are integrated by the middleware shared by both the networks, so that data can be seamlessly accessed by the upper layer irrespective of their source. Now, the upper layer, which is cloud computing platform, can provide a different type of services like storage, computation, analytical services and so on, using the different service components offered by cloud computing environment. The services compose of different business processes like health monitoring, environment monitoring, surveillance, smart transportation etc., which are seamlessly accessed by consumers irrespective of time and place.

In figure 5, the raw data captured by the sensing layer are transferred to middleware and from there to cloud computing infrastructure. The different layers of cloud computing, process the raw data. Now this processed data is sent to upper layer to provide different services, which end user can access through Standard Interface. Now, the IaaS of cloud exploits the massive cluster of servers at the back end. The operation of these clusters of servers is associated with consumption of a lot of energy in view of their working along with their cooling process. Again, proper energy management is also an important concern to report at the sensing layer. In Figure 5, the energy monitoring controller manages the energy of hardware at service level as well as operational level. In this perspective, this manuscript has proposed two techniques to monitor the energy management, which is at IaaS level and at sensing layer level. But during the optimization process of energy, one should not compromise with the QoS. Energy optimization along with the maintenance of QoS is today's need. The rest part of this manuscript has presented the graph-theoretic model for energy saving scheme at sensing layer level along with at IaaS level.

\subsection{Proposed algorithm}

\section{Step 1. [Initialization]}

Sensor nodes are distributed in a plane or in threedimensional space as per Hypothesis 1 .

Initially, $\lambda=0$ [Sensor nodes are in dormant state.]

Step 2. [Increasing the value of $\lambda$ ]

When $\lambda=\lambda_{1}$, consider a specific value of $\mathrm{x}$, say $\mathrm{x}_{1}$. With $\mathrm{x}_{1}$ as a center, construct a circle or sphere of radius ' $r$ ', where $r$ is a fixed positive real number. Join all the sensor nodes within the circle (sphere) and made them active and rest are made in the dormant state.

Step 3. [Finding the critical value of ' $\lambda$ ', say, $\lambda_{c}$ ]

IF the sensed value by the sensor meets the need of our requirement and no further sensing of data is needed then stop.

ELSE increase the value of $\lambda=\lambda_{1}<\lambda_{2}<\lambda_{3}<\lambda_{\mathrm{c}}$ so that we get a larger connected graph to meet our requirement.

Step 4. End.

\subsection{Explanation, justification and threat of proposed algorithm}

The proposed algorithm is based on the concept of partition of the plane or three-dimensional space into two parts namely active zone and passive zone. The sensor nodes at active zone are made active whereas the nodes at passive zone are made in the dormant state. The partition is made on the basis of random geometry graph concept. In this way, only a few parts of the plane or threedimensional space are made active to provide necessary raw data to upper layer with spending less energy as compared to make the whole plane or three-dimensional space active.

The major threat associated with this approach is, the selected zone may have a sensor node that all are out of order and thus may cause the overall failure of capturing the raw data. But in practical sense, the probability of such scenario is very less.

\section{Experimental setup \& result}

We evaluate the performance of our proposed approach through simulations. Here MATLAB, Release: R2013a software has been used for the purpose of simulation of sensor nodes in the plane. Let us now assume that the nodes are uniformly scattered in a $\mathrm{k} \times \mathrm{k}$ square space 
according to Poisson point process where the number of nodes is $\mathrm{n}$. For a given value of $\mathrm{r}$, we intend to find the critical value of $\lambda_{c}$ so that the random geometric graph $G$ $(n, r)$ has larger connected components. The experimental results are shown in Figure 6 for different values of $\lambda$ (Case (a) for $\lambda=200$, Case (b) for $\lambda=500$, Case (c) for $\lambda$ $=1000$, Case (d) for $\lambda=2000$, Case (e) for $\lambda=2500$ and Case (f) for $\lambda=5000$ ); where red dots are Active nodes and blue dot are hibernated nodes.

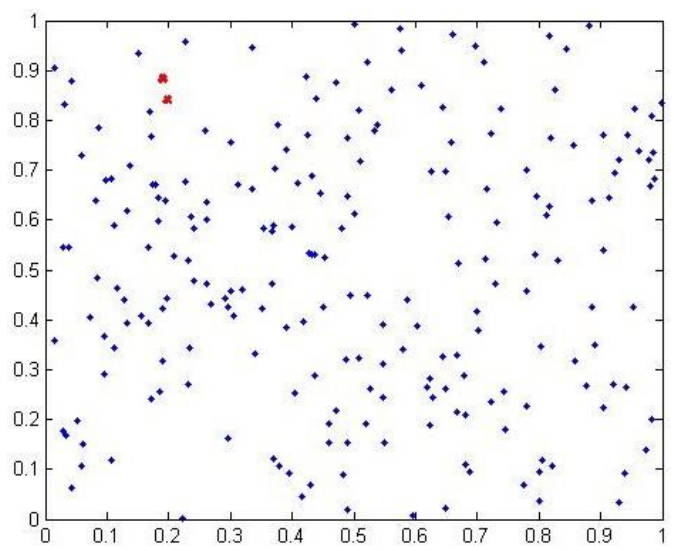

Figure 6: Case (a) for $\lambda=200$.

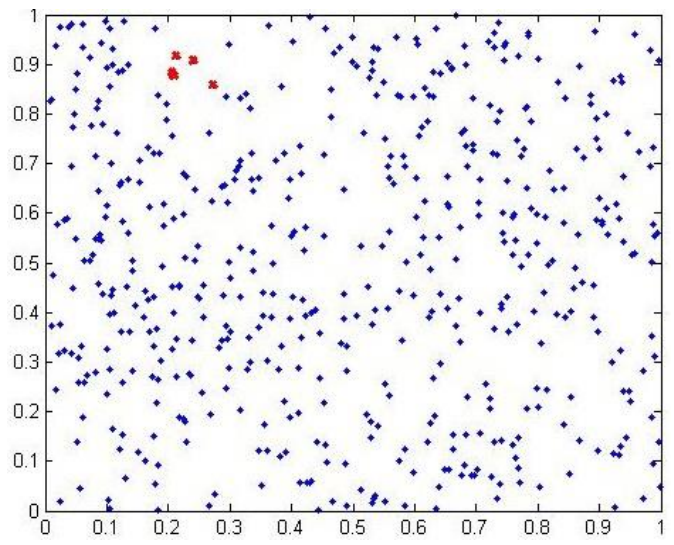

Figure 6: Case (b) for $\lambda=500$.

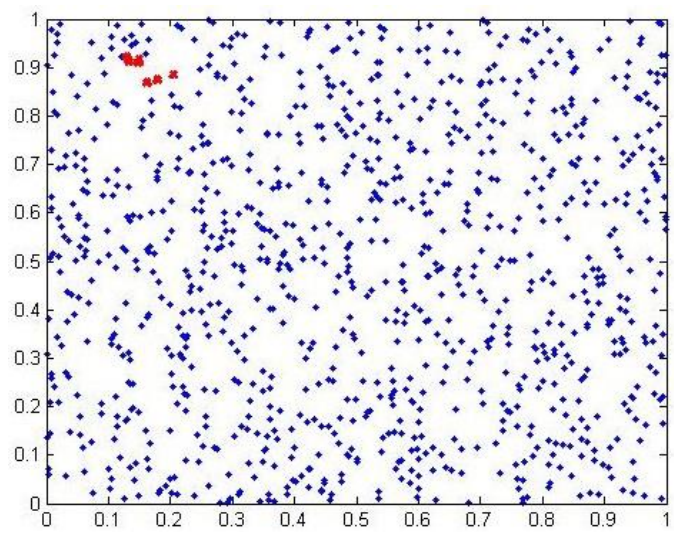

Figure 6: Case (c) for $\lambda=1000$.

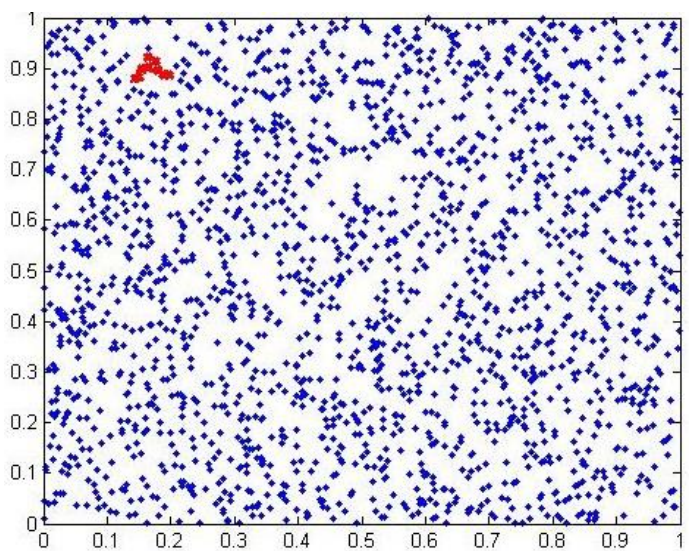

Figure 6: Case (d) for $\lambda=2000$.

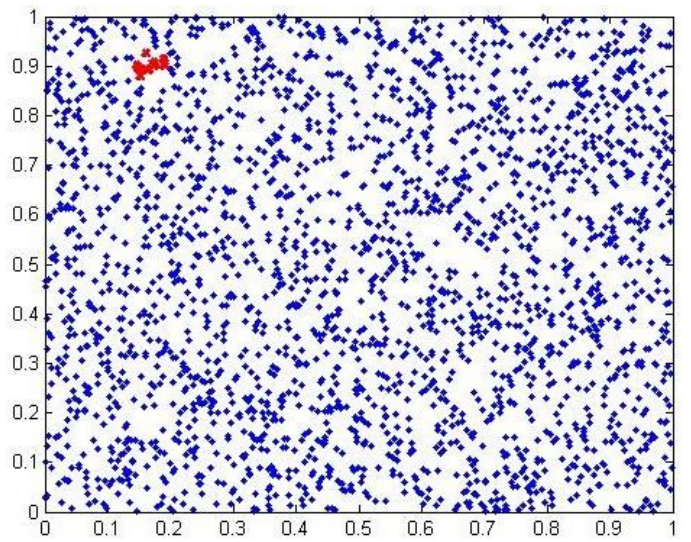

Figure 6: Case (e) for $\lambda=2500$.

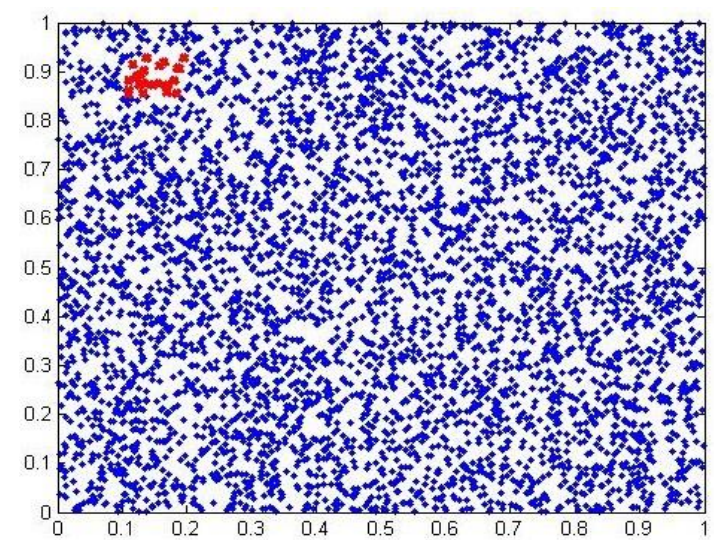

Figure 6: Case (f) for $\lambda=5000$.

Points arranged as per Poisson point process for different values of $\lambda$.

In Case (a) of Figure 6, few nodes are in active state (shown using red color), whereas the rest of the nodes are in the dormant state (shown using blue color) for $\lambda=100$. The experimental result points out that only those nodes are made active which are needed for communication and the rest of the nodes are made in dormant state in order to save energy. Now if a few nodes are active then it is not convenient for establishing efficient communication 
between the nodes. That is why it is needed to increase the value of $\lambda$ and to get a critical value of $\lambda_{c}$ for sufficient enough active nodes.

Based on the above experiments, the critical value of $\lambda_{\mathrm{c}}$ using the interpolation method is shown in Figure 7. We observe that as $\lambda$ increases, the nodes come very close to each other and hence more nodes are connected with one another. Thus there is a critical value of $\lambda_{c}$ for which $\mathrm{G}(\mathrm{n}, \mathrm{r})$ has a larger connected component within, which is shown in Figure 7.

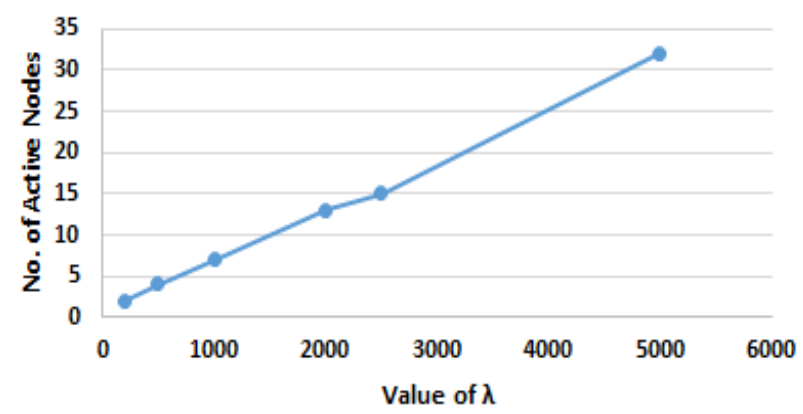

Figure 7: Threshold value of $(r, n)$ for which $G(n, r)$ has larger connected components

\section{Conclusion}

In the current scenario, IoT uses static graph which consumes more energy as it makes active every node all the time. Energy is only used in an active state while the dormant state consumes very less energy. Thus, keeping this in mind, we have proposed an energy efficient architecture based on SOA with their algorithm. In the proposed architecture, only the required number of nodes become active while another stay in dormant state, so that the energy consumption will reach at a minimal level. Energy being a precious resource, always demands optimization. Hence, the issue of drainage of energy in IoT can be well addressed by the implementation of above said energy-efficient architecture.

\section{References}

[1] Bui N., A. Georgiadis, M. Miozzo, M. Rossi, X. Vilajosana (2011). SWAP Project: Beyond the state of the art on harvested energy-power wireless sensors platform design. Proceedings of 2011 IEEE International Conference on Mobile Ad-Hoc and Sensor Systems, Valencia, Spain. https://doi.org/10.1109/MASS.2011.97

[2] Sung J., Lopez T.S., and Kim D. (2007). The EPC Sensor Network for RFID and WSN Integration Infrastructure. Proceedings of the 5th Annual IEEE International Conference on Pervasive Computing and Communications Workshops, New York, USA. https://doi.org/10.1109/PERCOMW.2007.113

[3] Giusto D., Iera A., Morabito G., Atzori L. (2010). The Internet of Things, Springer. ISBN: 978-1-44191673-0.

https://doi.org/10.1007/978-1-4419-1674-7
[4] Biswas, A. R., \& Giaffreda, R. (2014). IoT and cloud convergence: Opportunities and challenges. IEEE World Forum on Internet of Things (WF-IoT), pp. 375-376. https://doi.org/10.1109/WF-IoT.2014.6803194

[5] Atzori L., Lera A., Morabito G. (2010). The Internet of Things: A Survey. Computer Networks, Vol. 54, No. 15, pp. 2787-2805. https://doi.org/10.1016/j.comnet.2010.05.010

[6] Vermesan O., Friess P., Guillemin P., Gusmeroli S., Sundmaeker H., Bassi A., Jubert I. S., Mazura M., Harrison M., Eisenhauer M., and Doody P. (2011). Internet of Things - Global Technological and Societal Trends. Capter: Internet of Things Strategic Research Roadmap, River Publishers Series in Communications, pp. 9-52. PMid:20184663

[7] Kranz, M., Roalter, L. and Michahelles, F. (2010). Things that Twitter: social networks and the Internet of things. What can the Internet of Things do for the Citizen (CIoT) Workshop at The Eighth International Conference on Pervasive Computing (Pervasive 2010).

[8] Liu H., Bolic M., and Nayak A. (2008). Taxonomy and Challenges of the Integration of RFID and Wireless Sensor Networks. Journal of IEEE Network, Vol. 22, No. 6, pp. 26-35. https://doi.org/10.1109/MNET.2008.4694171

[9] Vermesan, O. and Friess, P. (2014). Internet of Things - From Research and Innovation to market Deployment. River Publishers Series in Communications, Denmark, pp. 07-112

[10] Gartner (2014). Internet of things will transform the data center. Research Report by IoT - Internet of Things. Available online at: https://iot.do/gartnersays-internet-things-will-transform-data-center2014-03

[11] Miorandi D., Sicari S., Pellegrini F. D., and Chlamtac I., (2012). Internet of things: Vision, applications and research challenges. Ad Hoc Networks, vol. 10, no. 7, pp. 1497-1516. https://doi.org/10.1016/j.adhoc.2012.02.016

[12] Peng Y., He U. and Choi J. (2012). Energy Efficient Hop Length Optimization for Laterally Connected Wireless Sensor Networks. Proceedings of IoT workshop, Berlin Germany. https://doi.org/10.1007/978-3-642-32427-7_8

[13] Pujolle, G. (2006). An autonomic-oriented architecture for the internet of things. IEEE International Symposium on Modern Computing, JVA'06, pp. 163-168. https://doi.org/10.1109/JVA.2006.6

[14] Jammes, F. and Smit, H. (2005). Service-Oriented Paradigms in Industrial Automation. IEEE Trans. Industrial Informatics, vol. 1, no. 1, pp. 62-70. https://doi.org/10.1109/TII.2005.844419

[15] De Souza L.M.S., Spiess P., Guinard D., Kohler M., Karnouskos S., and Savio D. (2008). SOCRADES: A Web Service Based Shop Floor Integration Infrastructure. Proceedings of Internet of Things Conference (IoT '08), Switzerland, pp. 50-67. https://doi.org/10.1007/978-3-540-78731-0_4 
[16] Priyantha N. B., Kansal A., Goraczko M., and Zhao F. (2008). Tiny Web Services: Design and Implementation of Interoperable and Evolvable Sensor Networks. Proceedings of 6th ACM Conf. Embedded Network Sensor Systems, pp. 253-266. https://doi.org/10.1145/1460412.1460438

[17] Gurun S., Krintz C. and Wolski R. (2004). NWS Lite: A Light- Weight Prediction Utility for Mobile Devices. Proceedings of the 2nd international conference on Mobile systems, applications, and services, Boston, USA, pp. 2-11. https://doi.org/10.1145/990064.990068

[18] Sabbineni H., and Chakrabarty K. (2005). Locationaided flooding: An energy-efficient data dissemination protocol for wireless sensor networks. IEEE Transactions on Computers, Vol. 54, pp. 3646. https://doi.org/10.1109/TC.2005.8

[19] Adachi M., Hiraoka T., Komatsu N. (2008). A study on a resource allocation algorithm for on-demand data center services. Proceeding of the 10th international conference on advanced communication technology - ICACT. pp. 295-300. https://doi.org/10.1109/ICACT.2008.4493765

[20] Lubin B., Kephart J. O., Das R., Parkes D. C. (2009). Expressive power-based resource allocation for data centers. Proceedings of $21^{\text {st }}$ international joint conference on artificial intelligence, IJCAI - ACM Digital Library. pp. 1451-1456.

[21] Wang, C., Bi, Z., \& Da Xu, L. (2014). IoT and Cloud Computing in Automation of Assembly Modeling Systems. IEEE Transactions on Industrial Informatics, Vol. 10, Issue 2, pp. 1426-1434. https://doi.org/10.1109/TII.2014.2300346

[22] Duroc Y. and Andia, V. G. (2014). Towards Autonomous Wireless Sensors: RFID and Energy Harvesting Solutions. Internet of Things Challenges and Opportunities, Springer, Switzerland. https://doi.org/10.1007/978-3-319-04223-7_1

[23] Yamini B. and Selvi D. V. (2010). Cloud virtualization: A potential way to reduce global warming. IEEE International Conference on Recent Advances in Space Technology Services and Climate Change (RSTS \& CC) Chennai, India, pp. 55-57. https://doi.org/10.1109/RSTSCC.2010.5712798

[24] Park K. and Valduriez P. (2011). Energy Efficient Data Access in Mobile P2P Networks, IEEE Transactions on Knowledge and Data Engineering. Vol. 23, No. 11, pp. 1619-1634. https://doi.org/10.1109/TKDE.2010.194

[25] Liu D., Zhang J., and Yu C. (2012). Energy efficient massive data dissemination protocols for the International Network of Things. Frontiers in Computer Education, pp. 627-634. https://doi.org/10.1007/978-3-642-27552-4_84

[26] Yuan W, Nahrstedt K. (2003). Energy-efficient soft reat-time CPU scheduling for mobile multimedia systems. ACM SIGOPS Operating Systems Review, Vol. 37, Issue 5. https://doi.org/10.1145/1165389.945460
[27] Dzmitry K., Sisay T. A., Fabrizio G. (2013). eSTAB: Energy-Efficient Scheduling for Cloud Computing Applications with Traffic Load Balancing. IEEE International Conference on Green Computing and Communications and IEEE Internet of Things and IEEE Cyber, Physical and Social Computing, Beijing, China, pp. 07-13. https://doi.org/10.1109/GreenCom-iThingsCPSCom.2013.28

[28] Hwang K., Fox G. C. and Dongarra J. J. (2012). Distributed and Cloud Computing From Parallel Processing to the Internet of Things. Morgan Kaufmann (An imprint of Elsevier), USA.

[29] Zhang L., Wang X., and Dou W. (2004). A KConnected Energy-Saving Topology Control Algorithm for Wireless Sensor Networks. Proceedings of the $6^{\text {th }}$ International Workshop on Distributed Computing, Kolkata, India. https://doi.org/10.1007/978-3-540-30536-1_57

[30] Jin J., Gubbi J., Lou T., and Palaniswami M. (2012). Network Architecture and QoS Issues in the Internet of Things for a smart city. Proceedings of International Symposium on Communications and Information Technologies, Gold Coast, QLD. https://doi.org/10.1109/ISCIT.2012.6381043

[31] Vasseur J. P. and Dunkets A. (2010). Interconnecting Smart Objects with IP: The Next Internet. Morgan Kaufmann (An imprint of Elsevier), USA.

[32] Estrin D., Govindan R., Heidemann J., and Kumar S., (1999). Next century challenges: scalable coordination in sensor networks. Proceedings of the $5^{\text {th }}$ annual ACM/IEEE International Conference on Mobile Computing and Networking, Seattle, Washington, USA. https://doi.org/10.1145/313451.313556

[33] Hui J. W. and Culler D.E. (2008). IP is dead, long live IP for wireless sensor networks. Proceedings of the 6th ACM Conference on Embedded Network Sensor Systems, New York, USA. https://doi.org/10.1145/1460412.1460415

[34] Penrose M., (2003). Random Geometric Graphs. Oxford University Press, USA.

https://doi.org/10.1093/acprof:oso/9780198506263. 001.0001 
